\title{
Gastroscopy combined with intragastric photography
}

\author{
D. D. GIBBS \\ B.A., D.M., M.R.C.P. \\ Consultant Physician
}

\author{
D. J. PARRY \\ B.Sc., M.B., M.R.C.P. \\ Consultant Physician
}

\begin{abstract}
Good Hope General Hospital, Sutton Coldfield, Warwickshire
\end{abstract}

\begin{abstract}
Summary
This paper relates experience in the use of the Olympus GTF fibrescope and intragastric camera. One hundred and eighty-four examinations were made in 157 patients.

The instrument was usually easy to introduce into the stomach and examinations were well tolerated. The youngest patient examined was aged 16 years and the oldest 86 . Fibroscopy in the elderly seldom presented difficulties.

This model(GTF) was particularly suited to examination of the mid-stomach and antrum, the view often extending to the pylorus. The range proximal to the angulus on the lesser curve and posterior wall was often limited.

Intragastric photographs of high quality could be taken; they enhanced the value and added to the interest of examinations. Serial pictures, taken at intervals, provided evidence of alterations in the appearances of gastric lesions.

Gastric endoscopy is complementary to radiological examination of the stomach. Its use often increases diagnostic accuracy and understanding of gastric diseases. Within the limits of design of a particular instrument, fibreoptic apparatus renders the procedure simple and efficient.
\end{abstract}

\section{Introduction}

The use of fibreoptics and the application of improved photographic techniques have led to a rewakening of interest in gastric endoscopy during the past few years. A bewildering variety of instruments is now available. Each instrument has been constructed with emphasis on one or more particular features and there is no universal gastroscope suitable for all requirements. The characteristics of the stomach, with its asymmetry, flabbiness and variability in shape and motility, are such that it is doubtful whether a single all-purpose tool could ever be constructed.

Previous articles by Hadley (1965) and Williams, Truelove \& Gear (1968) have related experience in this country with the gastrocamera (Olympus model GT5), and a fibrescope with special attachments which allow biopsy and cytology under direct vision in addition to photography with a recording camera attached to the proximal end of the instrument (Olympus model GFB). The present article is concerned with our experience in the past 2 years in the use of a fibrescope, which combines the facility for direct inspection of the stomach with an opportunity to take photographs by means of a miniature intragastric camera set alongside the viewing lens of the fibrescope (Olympus model GTF). The camera is independent of the fibrescope and the angle of the photographic field is slightly wider $\left(80^{\circ}\right)$ than that of the viewing lens of the fibrescope $\left(60^{\circ}\right)$.

\section{The instrument}

The fibrescope used has been an Olympus model GTF made in Tokyo. After air insufflation down a side channel the endoscopic view is obtained though a flexible bundle of glass fibres, at a magnification of $\times 7$. Two cold sources of light set near the tip of the instrument provide illumination. One bulb gives light for visual observation and a separate bulb in the same holder issues a flash lasting 1/15th second, for the purpose of photography. The intragastric camera has a fixed focus and contains neither iris nor shutter. The stomach acts as a dark room and a photograph is taken by activating the short duration flash without need of a shutter mechanism. Three settings on a control unit attached to the instrument allow variations to be made in the intensity of the flash, and this is adjusted according to the distance of the part of the stomach to be photographed, from the camera lens.

The film, measuring $5 \times 300 \mathrm{~mm}$, is contained in a metal casette and is loaded after unscrewing the tip of the instrument and attaching the free end of the reel to a metal lead. Thirty-two exposures are available from the film, each frame being drawn into position by operating the film lead from the 
proximal end of the fibrescope. During a period of viewing through the fibrescope one frame of film is exposed and the film must be advanced a further frame before photography. A few frames are wasted in this way, but usually sufficient remain to meet photographic requirements for examinations on two or more separate patients, using one reel of film.

Both the viewing lens of the fibrescope and the camera lens, are set in the side of the instrument, the view being at right angles to its long axis. The model (GTF) upon which our experience is based is not provided with a mechanism for altering the angulation of the tip of the instrument, a device which has been incorporated into a modified fibrescope (GTF-A). This means of controlling the direction of view by up or down movement is likely to be of particular value in increasing its range in the body of the stomach, on the lesser curve aspect. It was claimed that improvement had been derived at the cost of some reduction in flexibility, a quality particularly useful for viewing the distal antrum and pylorus (Morrissey, Tanaka \& Thorsen, 1967). But the versatility of the GTF-A instrument allowing views of both the most proximal and distal parts of the stomach has recently been confirmed in this country (Cockell \& Hawkins, 1969).

\section{Method of examination}

Most patients spent one night in hospital prior to examination; a few were examined as out-patients, and others were examined during longer periods of admission to hospital involving other investigations. All patients submitted to fibroscopy had previously been examined by a barium meal.

Fluid and food were withheld from 22.00 hours before the examination. Premedication with Omnopon and scopolamine, or with promethazine and atropine, was given approximately $1 \mathrm{hr}$ before each examination. The patient was given an amethocaine lozenge to suck for $15 \mathrm{~min}$ before the examination, and the pharynx was then sprayed with xylocaine spray, $1 \%$. Additional pharyngeal anaesthesia was provided by local application of viscous lignocaine (20 $\mathrm{mg}$ in $1 \mathrm{ml}$ ).

The instrument was passed with the patient lying in the left lateral position, the head being held by an assistant in a position of moderate extension. The tip of the instrument was placed over the tongue and guided in the right direction by the examiner's finger. Once cricopharyngeal resistance had been overcome the tube was gently introduced into the stomach and its passage could be assisted by swallowing movements by the patient. Unnecessary apprehension was usually dispelled if patients were assured beforehand that the examination involved only mild discomfort and no pain, and that it in no way impeded breathing.

Passage of the tube through the cardia was judged by the length of the tube that had been introduced (marked at $40 \mathrm{~cm}$ ) and was sometimes indicated by a feeling of slight resistance. After advancing the tube a further short distance the stomach was inflated and the light for visual observation switched on.

In the patient whose gastric anatomy had not been altered by previous surgery, the angulus could usually be identified rapidly and without difficulty, so that orientation of the view within the stomach was soon achieved. The wide angle view obtained contributed greatly to ease of interpretation and contrasted with the more limited area of the stomach visible through the lens systems of conventional gastroscopes. The examining light was visible in most patients through the anterior abdominal wall and could also be used to indicate the position of the instrument's tip.

The patient was usually examined supine as well ass on the left side and other adjustments in the patient's position were made to facilitate viewing of particular areas. As wide an area of the stomach as possible was examined within the limitations imposed by this particular instrument. The angulus, a considerable area on the greater curve aspect of the body, and most or all of the antrum often including the pylorus were seen routinely. In many patients only a limited view was obtained of the lesser curve of the stomach proximal to the angulus, and the cardia remained 'blind' to this instrument.

\section{Patients examined}

Examination has been undertaken in 157 patients and repeated on twenty-seven occasions in order to assess changes in appearances; eight of the examina-

\section{Explanation of Plate 1}

Fig. 1. View in the antrum showing a carcinoma with a rolled proximal edge and infiltrating distally in the entire circumference.

FIG. 2. Fungating carcinoma showing streaks of haemorrhage along its inferior border.

Fig. 3. Irregular carcinomatous mass involving extensively the lesser curve aspect of the body of the stomach.

FIg. 4. Superficial carcinoma on the lesser curve proximal to the angulus.

FIG. 5. Chronic benign gastric ulcer causing some contraction in the medial wall of the antrum and surrounded by oedematous mucosal folds.

FIG. 6. Penetrating gastric ulcer.

Fig. 7. Shallow gastric ulcer at the angulus on the lesser curve. The mucosa is diffusely abnormal containing small areas of haemorrhage and adherent mucus.

Fig. 8. Ulcer in the distal part of the antrum on the greater curve aspect. 

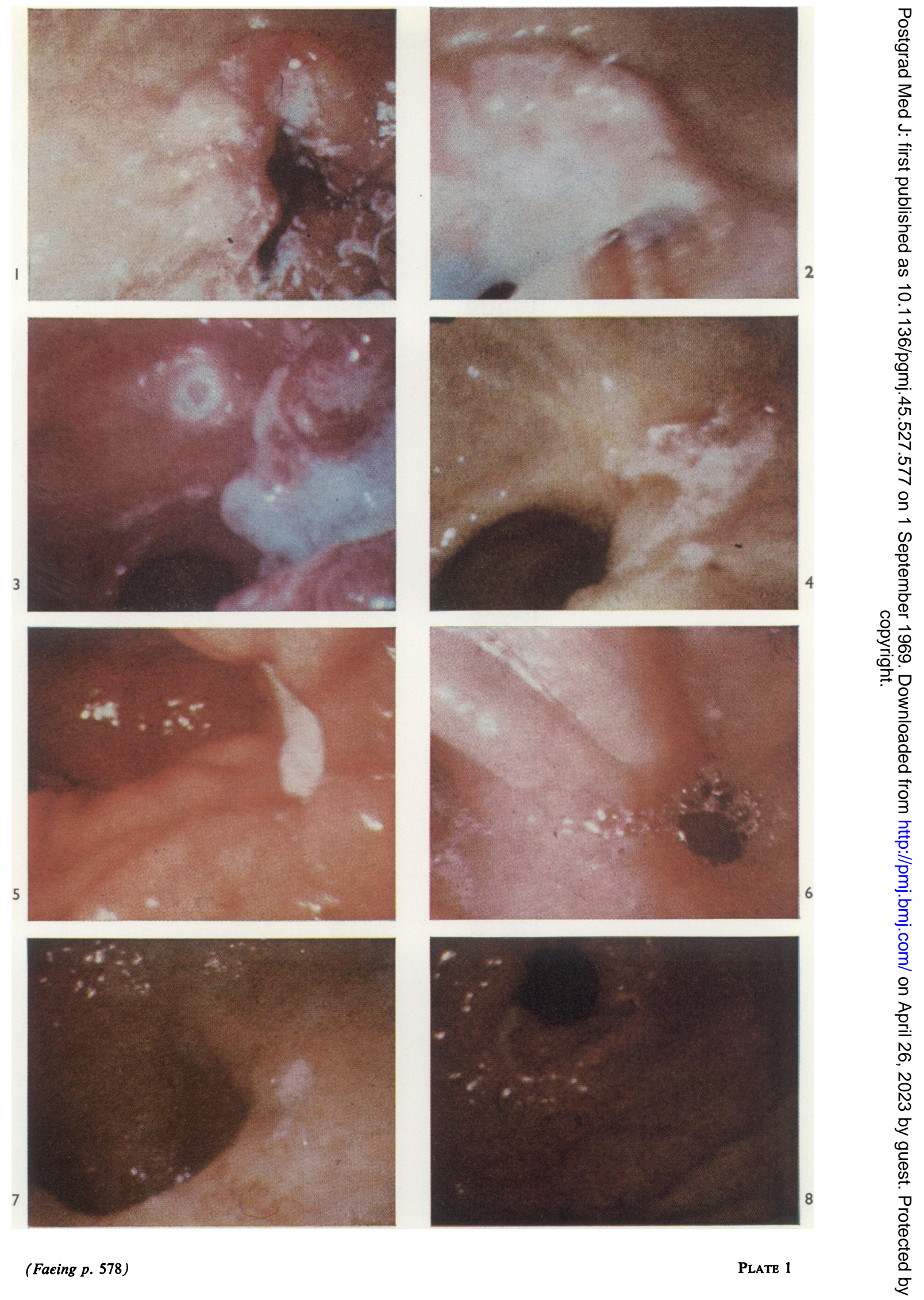
tions were failures and 176 were successful. Explanations for the failures were as follows: in four patients the tip of the fibrescope failed to negotiate the pharynx and cricopharyngeal area because of voluntary resistance and fear by the patient, despite detailed explanation and reassurance; macroglossia and abnormal tethering of the tongue impeded introduction of the tube in one patient, who proved to have amyloidosis; two patients had narrowing of the oesophagus near the cardia, probably as a result of oesophagitis; and in one elderly patient with kyphosis the tube was held up in the mid-oesophagus.

In this series there were no complications known to be directly related to the examinations, other than some soreness felt in the throat for a day or two. One elderly patient with a large lesser curve ulcer, which was not visualized by fibroscopy, was admitted to hospital 3 days after the examination, with a gastric perforation.

The ages of patients examined ranged from 16 to 86. Elderly patients were well represented, twentytwo being 70 or over and five were octogenarians.

The results of 176 successful examinations carried out on 149 patients are analysed and discussed mainly in relation to the diagnostic conclusions derived in individual cases. Barium radiology, fibroscopy and gastric photography were undertaken in all patients, but were supplemented by other standard diagnostic procedures in most cases. Diagnosis is, of course, most securely founded in patients when there is demonstrable abnormality; more tentative conclusions have to be drawn after negative investigations on patients with upper abdominal symptoms. The following categories provide a framework in which to present the results and discuss their relevance:

(1) Patients with normal stomachs on X-ray in whom the endoscopic appearances were also normal (thirty-seven).

(2) Patients with carcinoma (eleven).

(3) Patients with chronic peptic ulcers shown to be present on the lesser curve of the body of the stomach by X-ray examination (forty).

(4) Patients found to have benign ulcers in the antrum or pre-pyloric region (eight).

(5) Patients with superficial mucosal ulceration (seven).

(6) Patients with benign lesions other than peptic ulcers-including those with diffuse mucosal changes interpreted as gastritis (eighteen).

(7) Patients who had previously undergone gastric surgery (twenty-eight).

\section{Results}

\section{Normal gastric appearances}

Thirty-seven patients were considered to show normal appearances, on fibrescopic examination.
In the context of normal radiographic findings and from the results of other routine investigations, there was no reason to conclude that gastric disease was a cause for their symptoms. Seventeen of these patients were thought to have disturbances of bowel motility to explain their symptoms, twelve had duodenal ulceration or deformity on X-ray, and others had oesophagitis or non-gastric abdominal malignancy.

The negative findings were sometimes helpful in directing investigation to other parts of the alimentary tract. In some individuals the knowledge that their stomachs had been subjected to detailed visual and photographic scrutiny may have contributed to effectiveness in symptomatic management.

The area of stomach visualized varied. The angulus and mid-portion of the stomach were examined satisfactorily in all these patients and a good view was obtained into the antrum although not always as far as the pylorus itself. In more than half this group of patients the pylorus was identified as a circular structure which opened and closed, often with retrograde emissions of a few bubbles of bile-stained fluid. In these patients comprehensive examination of the distal antrum was confidently achieved. In a proportion of patients the direction of the distal antrum prevented full access into it and a prepyloric lesion was not excluded; in others, especially when there was little or no peristaltic activity, the exact location of the pylorus could not be determined. At times the tip of the fibrescope probably entered the duodenum although no endoscopic information of interest was forthcoming.

\section{Carcinoma}

Eleven patients proved to have carcinoma of the stomach (Figs. 1-4). Eight were confidently diagnosed radiologically. In two of these patients the growths could not be seen by fibroscopy because of their proximal situations, but in the other six the endoscopic appearances fully supported the radiological diagnoses. In two patients the $\mathrm{X}$-ray appearances were suspicious of malignancy and these suspicions were confirmed by the endoscopic features. In one patient, admitted because of haematemesis, a barium meal was considered normal, but fibroscopy showed a shallow irregular ulcer which looked, and proved to be, neoplastic (Fig. 4).

The number of patients examined is too limited for generalizations to be valid, but the contribution of gastric endoscopy as one element in a threepronged investigational approach to carcinomabarium radiology followed by cytology and endoscopy-is well established.

The model (GFB) described by Williams et al. (1968) is likely to be especially valuable when 
malignancy is suspected, because it provides an opportunity to obtain histological and cytological information at the same time as a lesion is viewed and photographed. Further experience will be necessary with this instrument before its full range and efficiency in producing diagnostic information in patients with gastric malignancy can be known.

\section{Lesser curve gastric ulcer shown on $X$-ray film}

Forty patients were examined after radiological demonstration of an ulcer niche on the lesser curve aspect of the stomach, usually a short distance proximal to the angulus, but in some cases high in the stomach. Sixteen re-examinations were made, usually on one occasion in the same individual, but exceptionally three or more examinations were undertaken, separated by intervals of treatment. In twenty-four patients $(60 \%)$ the ulcer was seen and photographed using the GTF instrument, but the ulcer-bearing area of stomach proximal to the angulus, often on the posterior wall, could not be brought into view in sixteen patients $(40 \%)$. The high failure rate for these lesions should be reduced by the mechanism allowing control of the tip of the fibrescope which is incorporated into the modified GTF-A instrument.

When an ulcer lay within range of the instrument an excellent view was obtained and precise analysis of its macroscopic characteristics was possible, by direct vision and in photographs (Figs. 5-7). Features such as the sharpness of the edges, the prominence of a hyperaemic halo, the shape and character of the base could be assessed in photographs. An indication of depth was sometimes not conveyed as effectively as that provided by the degree of penetration shown radiographically. Commonly there were diffuse abnormalities in the gastric mucosa of ulcer-bearing stomachs, particularly in the form of small areas of mucosal haemorrhage and contact bleeding, which often occurred as a result of gentle instrumentation (Fig. 7).

Nearly half the patients shown to have chronic peptic ulcers were treated surgically. In the remainder, the response to medical treatment was followed either by further X-ray examinations or by fibroscopy. In eight patients one or more re-examinations were carried out to assess the degree of healing that had occurred in the ulcer. These patients did not regard such serial examinations as a great ordeal and the information derived was valuable in guiding management policy. Pictorial comparisons provided accurate evidence on the stage of healing (Figs. 9-14).

\section{Benign antral ulcers}

In eight patients an ulcer was identified in the antrum or pre-pyloric region and in three patients re-examinations were undertaken after periods of treatment. Three patients were treated by surgery and the ulcers found to be benign; in the remainder, healing was followed either by means of X-ray examinations or by fibroscopy. Endoscopic identification of an ulcer in the distal stomach was helpful in deciding on the management of several of these patients. A small peptic ulcer was sometimes associated with considerable distortion and narrowing in the distal antrum, when examined radiologically; moreover the ulcer crater itself was often poorly defined in X-ray pictures. Such disturbances in motility which sometimes accompany ulcers in the distal antrum may lead to strong suspicions of malignancy when radiological evidence alone is used. A view of the relevant area by fibroscopy may suggest that the lesion responsible for the distortion is a small peptic ulcer, and once seen and photographed such a lesion can be available for reexamination to determine response to treatment (Fig. 8).

Superficial ulcers visible by fibroscopy but not demonstrated on $X$-ray examination

In seven patients the presence of superficial ulcers was suggested by the endoscopic appearances. In two there were minute irregularities visible in the barium X-rays which probably corresponded to mucosal lesions but diagnosis by this means was uncertain. In two patients multiple mucosal ulcers were present. Uncertainty in interpretation sometimes arose, particularly if surrounding hyperaemia or other mucosal changes were absent. The photographic appearances were sometimes helpful in confirming or refuting the presence of shallow small ulcers in the stomach.

Benign lesions, other than peptic ulcers, including diffuse mucosal changes

Eighteen patients were included in this category. In thirteen patients there were diffuse gastroscopic

\section{Explanation of Plate 2}

FIGS. 9-11. Intragastric photographs taken over a period of 10 weeks illustrating the stages of healing in a giant gastric ulcer, which occurred in a girl of 16.

Figs. 12-14. Healing of a giant lesser curve gastric ulcer followed in a patient of 81 , over a period of 6 weeks. A zone of hyperaemia, probably indicative of an active healing process, is illustrated in Fig. 13.

FIG. 15. A smooth swelling, situated proximal to the angulus, with streaks of pigmentation on the surface. The lesion proved to be an adenomyoma.

FIG. 16. Anterior wall of the mid-stomach showing multiple gastric polyposis. 

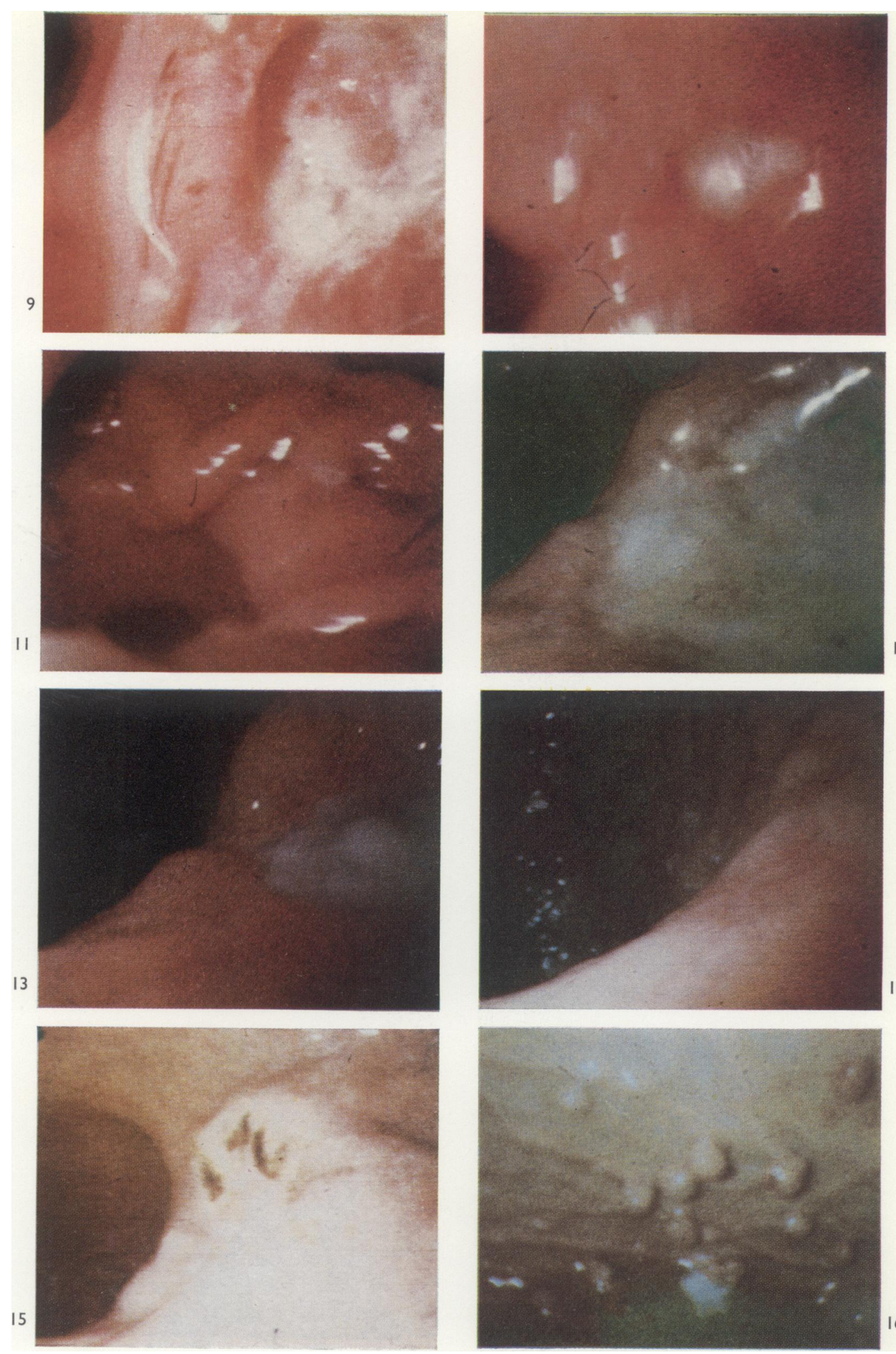
abnormalities which were interpreted as representing superficial or haemorrhagic gastritis, gastric atrophy or abnormally prominent rugal patterns. The difficulties of correlating such changes with clinical situations is appreciated, and an inability to obtain biopsy material is a disadvantage in such cases. Nevertheless, visual and photographic information proved diagnostically valuable in several individual patients. In one patient there were multiple haemorrhagic lesions in the mucosa accounting for clinically overt upper gastro-intestinal haemorrhage; barium meal was normal. In two patients haemorrhagic and oedematous changes were confined to the mucosa of the antrum and may have been responsible for symptoms, which included post-prandial discomfort and weight loss. Five patients were examined in whom barium radiology had shown abnormally deep rugal folds, particularly on the greater curve aspect of the body of the stomach; in two of these patients the X-ray findings suggested the additional possibility of gastric ulcers. The findings on fibroscopy in this group of patients varied, but the presence of ulceration was confidently excluded from the sites suspected from $\mathrm{X}$-ray examination.

In a woman of 42 , with a 2-year periodic history of epigastric pain after meals, an unusual lesion was identified and photographed on the lesser curve aspect of the antrum: it proved to be an adenomyoma (Fig. 15).

Two patients with multiple filling defects in the stomach discovered on X-ray were examined, and the presence of multiple polyposis confirmed (Fig. 16). Excellent photographic records of the polyps situated in the mid-stomach and antrum were made in each case. Although the polyps situated in the most proximal parts of the stomachs could not be visualized, the examinations provided valuable evidence to use for future comparison. As a premalignant condition, multiple gastric polyposis might be kept under accurate periodic review by means of photographic records, so that enlargement or changes in individual polyps could be detected to influence decisions in favour of surgery. In both patients examined in this study cytological information was also obtained and proved negative.

\section{The post-operative stomach}

Twenty-eight patients had previously undergone some form of gastric surgery; five of these were examined on more than one occasion. In five patients vagotomy and pyloroplasty had been carried out, and in two of these radiologically unsuspected gastric ulceration was discovered. In twelve patients gastroenterostomy had been performed either with a vagotomy or as part of a Polya resection. The gastric side of the stoma was seen in its entirety in seven cases and partially in five; an area of jejunal mucosa could usually be viewed but difficulty in manoeuvring the tip of the instrument limited examinations of the intestinal aspects of the stomas. In two patients stomal ulcers were identified and in a third there was extensive erosive gastritis with multiple superficial ulcers. Hyperaemia of the mucosa in the stomach remnant was common and small mucosal haemorrhages were sometimes visible.

Eleven patients had undergone Billroth I partial gastrectomy and with one exception the stoma was adequately visualized. Two patients had ulcers proximal to the stomas; in both these patients the ulcers were diagnosed easily, radiologically as well as fibrescopically. Healing was followed endoscopically in one patient by three interval examinations.

\section{Discussion}

The Olympus GTF fibrescope has proved simple and safe to use and, within a certain range inside the stomach, is an effective diagnostic instrument. Its flexibility contributes to ease of introduction and permits successful examinations in elderly patients, which would often be impossible using a semiflexible conventional gastroscope. The wide-angle view obtained within the stomach renders examination and orientation comparatively easy. The reliable interpretation of appearances requires some experience, but the technique of introduction and examination is learnt after only a short apprenticeship.

The instrument does not provide a view of the whole of the interior of the stomach. It is mainly an 'antroscope'. It allows excellent views of the distal and mid-stomach, but often gives little opportunity to see areas proximal to the angulus, particularly on the posterior wall. This instrument (GTF) has now been superseded by a fibrescope (GTF-A) which incorporates a mechanism by which the tip can be controlled and which has a comprehensive range within the stomach.

The provision of an intragastric camera set adjacent to the viewing lens of the instrument is a valuable asset and offers more than a luxury accessory. Colour transparencies taken with an intragastric camera are of excellent quality and retain sharp definition when projected. Not only is the quality superior to photographs taken with recording cameras which are set at the eyepiece of fibrescopes or gastroscopes, but the angle of photography is wider than that obtained in views taken through the viewing system. Such photographs, which are easily obtained during the examination, provide obvious opportunities for recording experience for self-instruction, teaching and inter-disciplinary discussions with radiologists and surgeons concerned 
in the investigation and management of the same patients.

The most interesting contribution of intragastric photography with this instrument is the ability to take serial photographs of certain gastric lesions in order to record changes that occur in response to treatment or during the course of time. The stages of healing of peptic ulcers in response to treatment may be accurately recorded in some patients. Comparative serial photographs of other lesions, such as mucosal abnormalities or adenomatous polyps, taken at long intervals of time, could prove of practical importance.

Since the earliest reports of experience with instruments designed for direct examination of the stomach, endoscopists have claimed success in defining certain lesions which have not been identifiable by other means. Even among the comparatively small number of patients who form the basis for this report, abnormalities such as superficial ulcers, mucosal bleeding, stomal ulceration and a superficial carcinoma have been discovered which were not suspected from radiological examinations. In addition to the discovery of radiologically unsuspected abnormalities in some patients, endoscopy provided a means of examining established lesions both three-dimensionally and in the context of changes in the mucosa and motility of other parts of the stomach.

By conventional gastroscopy, using an instrument with a lens system rather than fibreoptic bundles, these advantages could be gained only by investigators who were especially skilled and experienced. Our application of the GTF model Olympus fibrescope indicates that better views are obtained of the mid and distal stomach than with semiflexible instruments, and that the examination is easier to perform. Nevertheless, although attended by very little discomfort or risk to the patient, the investigation should not be considered an alternative to barium X-ray examination but as a valuable complementary method. Though greatly simplified by the use of such a sophisticated instrument, the examination remains something of an ordeal for a proportion of patients and this should temper enthusiasm for using gastric fibroscopy as a standard method of investigating all patients with upper abdominal symptoms. For many patients, however, our experience with this instrument indicates that it can provide valuable diagnostic information which sometimes helps to determine satisfactory management.

\section{Addendum}

Since writing this article our experience has extended to some 250 examinations. We have not found reasons to alter our general conclusions, but one case deserves mention. A patient with a small antral ulcer was re-examined after 2 weeks and the lesion appeared to have healed, as judged both by direct examination and from intragastric photographs. Examination by gastric exfoliative cytology was not carried out. Fifteen months later the patient presented with disseminated malignancy and a third fibroscopy showed a small ulcer at the same site as that identified in the original examination, the appearances being very similar. Temporary healing of peptic ulceration located in a small area of gastric neoplastic tissue has, in the past, been implied in other cases, from radiological evidence. The unfortunate sequence was recorded photographically in our case and illustrates the limitations of a single diagnostic approach in certain rare patients.

\section{Acknowledgments}

The GTF fibrescope incorporating a gastric camera was purchased by the Birmingham Regional Hospital Board for use in Good Hope General Hospital. We are grateful to our colleagues for referring patients for examination. It is a pleasure to acknowledge the interest and help of Dr C. I. Griffiths, Dr I. C. F. Riach and Dr F. E. McGuiness, in correlating the radiological findings with endoscopic information. Several generations of House Physicians have willingly assisted, and prepared patients for examination. Mr D. S. Wilkinson has devoted skill and care to enlarging the gastrophotos to make them suitable for publication.

Special thanks are due to Air-Shields (U.K.) Ltd for a generous grant which has made possible the publication in colour of the plates of gastric photographs.

\section{References}

Cockell, R. \& Hawkins, C.F. (1969) Gut, (In press).

HADLEY, G.D. (1965) The gastro-camera. Brit. med. J. 2, 1209.

Morrissey, J.F., TANaKa, Y. \& Thorsen, W.B. (1967) Gastroscopy. A review of the English and Japanese literature. Gastroenterology, 53, 456.

Williams, D.G., Truelove, S.C. \& Gear, N.W. (1968) Gastroscopy with biopsy under direct vision. Brit. med. J. 1, 535 . 